\title{
Versatile Fabrication of Self-Aligned Nanoscale Hall Devices using Nanowire Masks
}

Jianshi Tang, ${ }^{1 * \#}$ Guoqiang Yu, ${ }^{1}$ Chiu-Yen Wang, ${ }^{2}$ Li-Te Chang, ${ }^{1}$ Wanjun Jiang, ${ }^{1 \dagger}$ Congli He, ${ }^{1}$ and Kang L. Wang ${ }^{1 *}$

${ }^{1}$ Device Research Laboratory, Department of Electrical Engineering, University of California, Los Angeles, California, 90095, USA

${ }^{2}$ Department of Materials Science and Engineering, National Taiwan University of Science and Technology, Taipei City, Taiwan, 10607, Republic of China

"Present address: IBM Thomas J. Watson Research Center, Yorktown Height, New York, 10598, USA

${ }^{\dagger}$ Present address: Materials Science Division, Argonne National Laboratory, Argonne, Illinois, 60439, USA

*To whom correspondence should be addressed. E-mail: tjianshi@ucla.edu, wang@seas.ucla.edu

\section{Supporting Information}

S1. Preparation of the Ta/Co20 $\mathrm{Fe}_{60} \mathrm{~B}_{20} / \mathrm{MgO}$ film

S2. SEM images of VLS-synthesized $\mathrm{SnO}_{2}$ nanowires

S3. Fabrication of Ta/CoFeB/MgO nanoscale Hall device with smaller dimensions

S4. SEM images of a Ta/CoFeB/MgO nanoribbon cross

S5. Roughness analysis on the fabricated nanoribbon

S6. Current-induced magnetization switching in micron-scale Ta/CoFeB/MgO device 


\section{S1. Preparation of the $\mathrm{Ta} / \mathrm{Ta} / \mathrm{Co}_{20} \mathrm{Fe}_{60} \mathrm{~B}_{20} / \mathrm{MgO}$ film}

As the start material, stacks consisting of $\mathrm{Ta} / \mathrm{Co}_{20} \mathrm{Fe}_{60} \mathrm{~B}_{20} / \mathrm{MgO}(5 \mathrm{~nm} / 1.1 \mathrm{~nm} / 2 \mathrm{~nm}$ in thickness $)$ were deposited on a thermally oxidized $\mathrm{SiO}_{2} / \mathrm{Si}$ substrate at room temperature using magnetron sputtering in an AJA International Physical Vapor Deposition System. The deposition rates were $0.06 \mathrm{~nm} / \mathrm{s}, 0.03$ $\mathrm{nm} / \mathrm{s}$ and $0.003 \mathrm{~nm} / \mathrm{s}$ for Ta, $\mathrm{Co}_{20} \mathrm{Fe}_{60} \mathrm{~B}_{20}(\mathrm{CoFeB})$ and $\mathrm{MgO}$ at Ar pressure of $2 \times 10^{-3}$ Torr, respectively. Subsequently, another 1.5 nm-thick Ta layer was in situ deposited on top and then was exposed to radiofrequency $\mathrm{O}_{2} / \mathrm{Ar}$ plasma for $100 \mathrm{~s}$, forming a $\mathrm{TaO}_{\mathrm{x}}$ capping layer to prevent the degradation of the $\mathrm{MgO}$ layer in the ambient. The films were then annealed at $200{ }^{\circ} \mathrm{C}$ for $30 \mathrm{~min}$ to enhance their perpendicular magnetic anisotropy. 

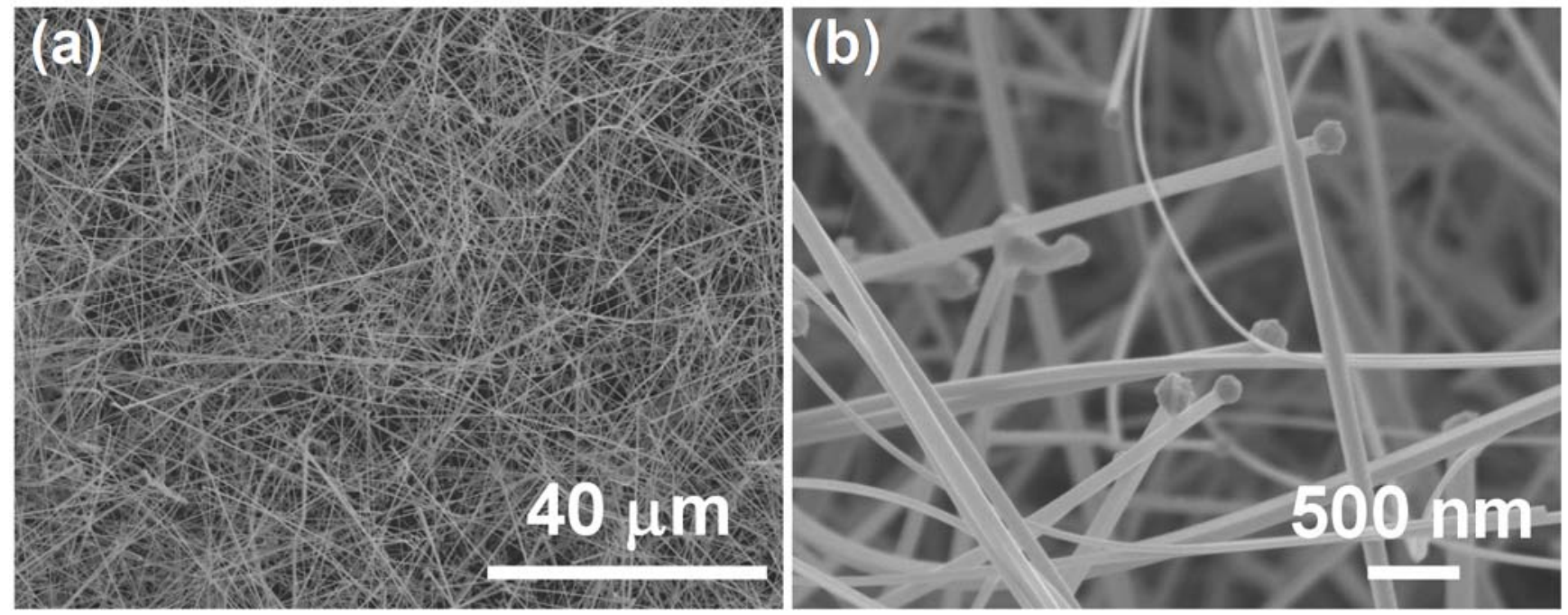

Figure S1. (a) SEM image of VLS-synthesized $\mathrm{SnO}_{2}$ nanowires on a bare Si substrate with lengths up to hundreds of micrometers. (b) Enlarged SEM image of the $\mathrm{SnO}_{2}$ nanowires, showing a typical diameter range from $50 \mathrm{~nm}$ to $200 \mathrm{~nm}$. 


\section{S3. Fabrication of Ta/CoFeB/MgO nanoscale Hall device with smaller dimensions}

There are a few major factors that could affect the dimension of the resulting nanoscale Hall device, especially the produced nanoribbon using nanowire as the etching mask. Firstly, the produced nanoribbon width is largely determined by the diameter of the mask nanowire: generally the smaller nanowire diameter, the smaller nanoribbon width (see Figure S2 below). Secondly, it could also be affected by the dry etching time: increased etching time could reduce the nanoribbon width. It should be noted that the etching time highly depends on the etching recipe, the thickness of the starting thin film material, and also the selectivity of the target material over the mask nanowire. In addition, in the fabrication of nanoscale Hall bar structure using the present method, the distance between two transverse contact pairs is determined by the metal deposition method (e.g., directional e-beam evaporation or conformal sputtering), as already shown in Figure 2 in the main text. Also, the shape of the mask nanowire could also play an important role: for example, circular nanowires are usually preferred over facet nanowires (triangular or rectangular nanowires, etc) as the etching and deposition mask for the purpose of making reliable electrical contacts and easy metal liftoff.


Figure S2. (a) SEM image of a fabricated Ta/CoFeB/MgO nanoribbon with self-aligned Ti/Al contacts using sputtering. (b) Enlarged SEM image of the Ta/CoFeB/MgO nanoribbon with self-aligned Ti/Al contacts, showing a nanoribbon width of about $40 \mathrm{~nm}$. 

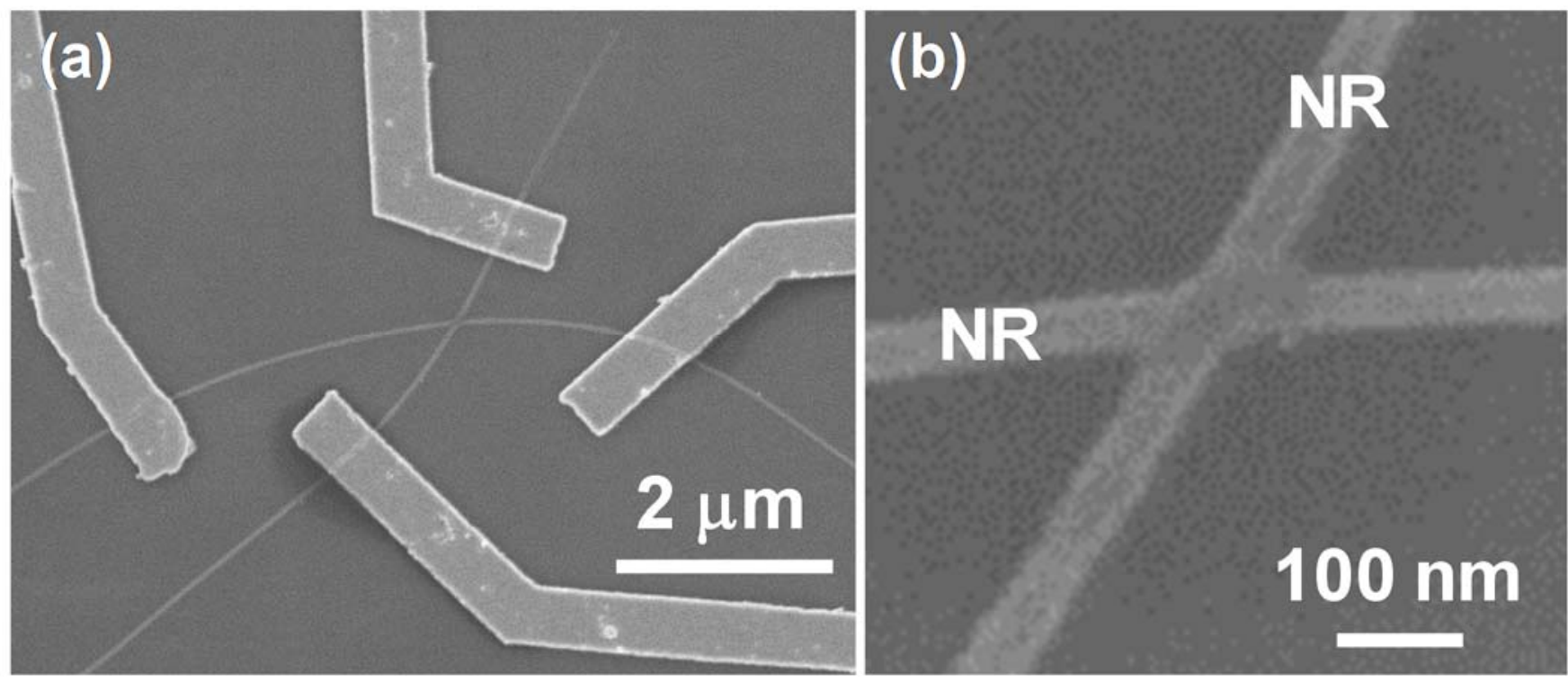

Figure S3. (a) SEM images of a Ta/CoFeB/MgO nanoribbon cross, whose morphology was transferred from two intersected nanowires as the etching mask. (b) Enlarged SEM images of the Ta/CoFeB/MgO nanoribbon cross, showing a width of about $40 \mathrm{~nm}$. This cross can also be used as a test structure for (anomalous) Hall effect measurement. 


\section{S5. Roughness analysis on the fabricated nanoribbon}

We have performed detailed physical characterizations of the quality of the resulting nanoribbon in comparison with the starting $\mathrm{Ta} / \mathrm{CoFeB} / \mathrm{MgO}$ film, and the results are shown in Figure S4 below. The as-grown $\mathrm{Ta} / \mathrm{CoFeB} / \mathrm{MgO}$ film had a very smooth surface morphology shown in Figure S4(a) with a root-mean-square (RMS) surface roughness of about $0.3 \mathrm{~nm}$ (see Figure S4(b)). After dry etching through nanowire mask and making self-aligned metal contacts to the resulting nanoribbon, the surface became a little rougher, as shown in Figures S4(c-d). The RMS surface roughness was slightly increased to about $0.9 \mathrm{~nm}$ (see Figure S4(e)). This is likely due to the presence of unavoidable PMMA resist residues during the e-beam lithography process to define metal contacts. However, the surface damage on the $\mathrm{Ta} / \mathrm{CoFeB} / \mathrm{MgO}$ stack (with only a few nanometers in thickness) was rather minor because the perpendicular magnetic anisotropy was preserved in the resulting nanoribbon, as already shown in Figure 3 in the main text.

Furthermore, the width variation along the nanoribbon length direction (i.e., mask nanowire axis direction) was extracted from the high-magnification AFM image and shown in Figure S4(f). The linewidth roughness (standard deviation) was estimated to be about $2.2 \mathrm{~nm}$, less than $2 \%$ of the nominal nanoribbon width (about $150 \mathrm{~nm}$ ). It should be pointed out that in the present method (nanowire lithography), the line-width roughness of the resulting nanoribbon could be affected by many factors, such as the surface roughness of the mask nanowire itself and the uniformity of the dry etching process as well as possible resist residues or contaminations near the nanoribbon edge, etc. For example, any surface defects of the mask nanowire could be directly transferred onto the resulting nanoribbon. In our earlier study of making graphene nanoribbon (Ref. [23] Xu, G.; Torres, C. M.; Bai, J.; Tang, J.; Yu, T.; Huang, Y.; Duan, X.; Zhang, Y.; Wang, K. L. Appl. Phys. Lett. 2011, 98 (24), 243118), it was found that the line-width roughness decreased with the graphene nanoribbon width, and it could degrade the device performance by introducing additional variation in transport properties. In our present study, the line-width roughness was relatively small, and hence apparently it had little effect on the measured 
magnetic properties, as evident by the preserved perpendicular magnetic anisotropy and sharp switchings shown in Figures 3-4 in the main text.
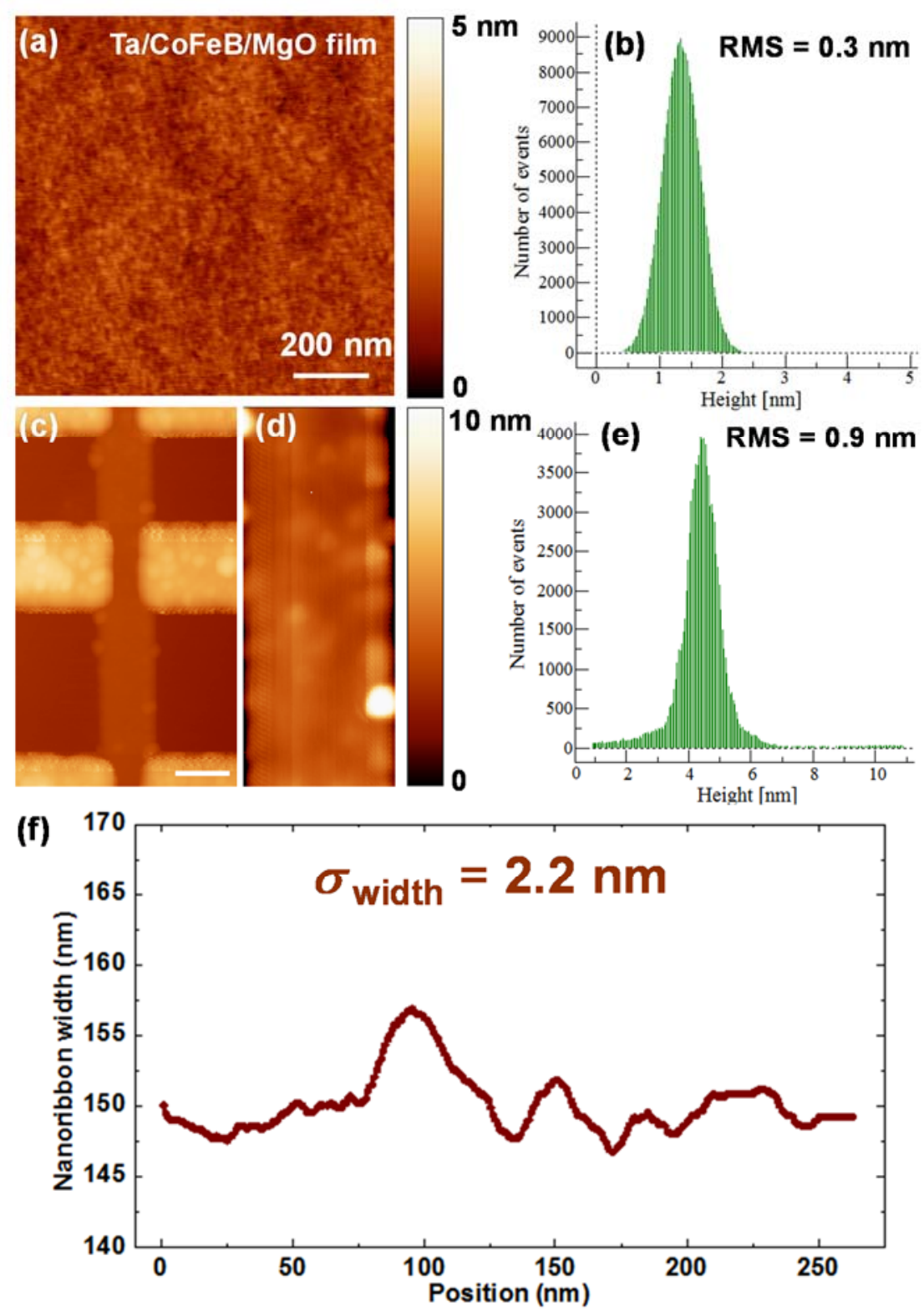

Figure S4. (a) AFM image of as-grown Ta/CoFeB/MgO film showing a smooth surface morphology.

(b) Surface roughness analysis of (a) shows a root-mean-square (RMS) roughness of 0.3 nm. (c) AFM image of a fabricated $150 \mathrm{~nm}$-wide Ta/CoFeB/MgO nanoribbon. The scale bar equals to $150 \mathrm{~nm}$. (d) High-magnification AFM image the nanoribbon, showing a slightly rougher surface than the original film. This is mainly due to the resist residue during the fabrication process. (e) Surface roughness analysis of (a) shows a root-mean-square (RMS) roughness of $0.9 \mathrm{~nm}$. (f) Extracted nanoribbon width along the mask nanowire axis direction. The line-width roughness is about $2.2 \mathrm{~nm}$. 
S6. Current-induced magnetization switching in micron-scale Ta/CoFeB/MgO device
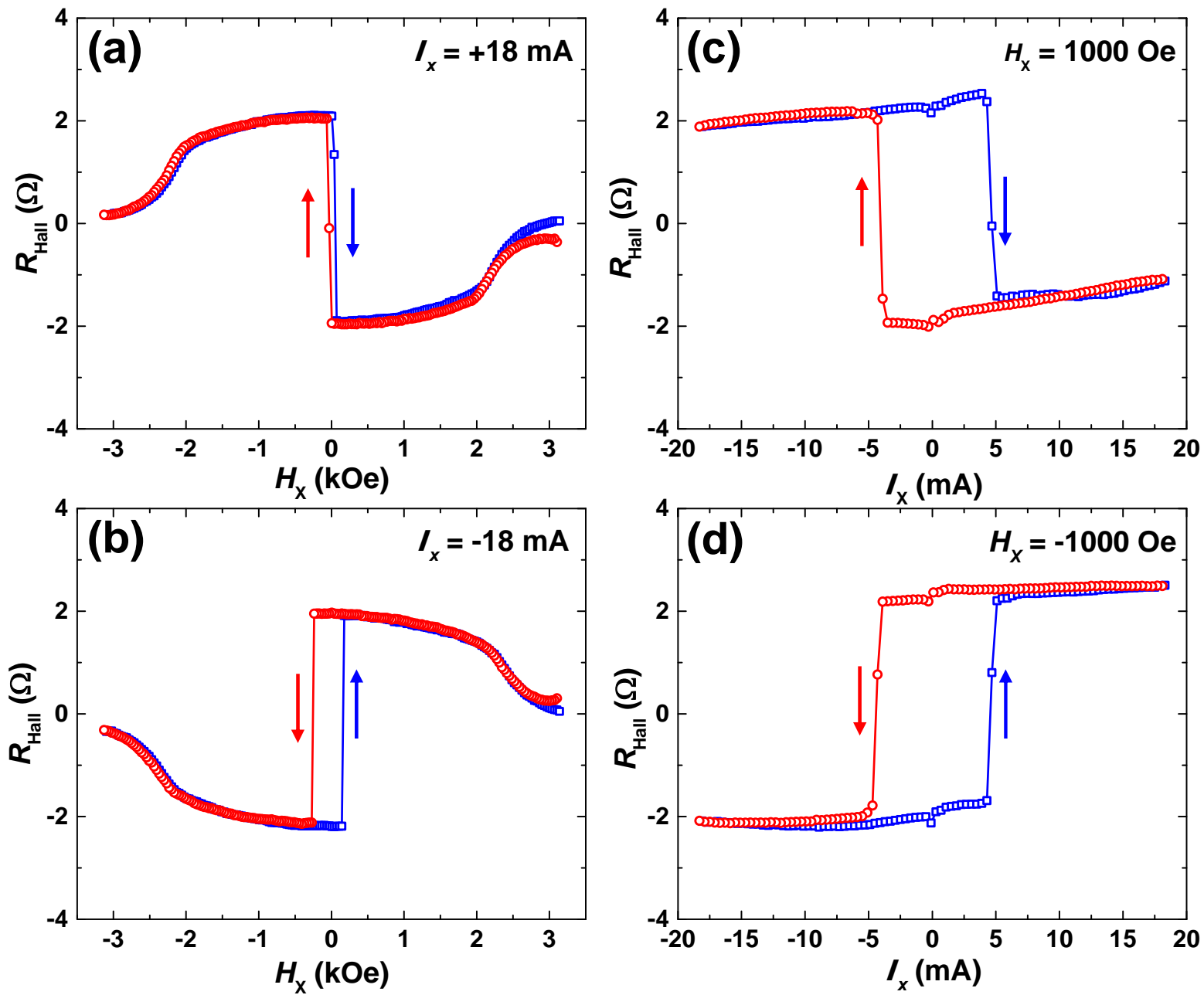

Figure S5. Current-induced magnetization switching in micron-scale Ta/CoFeB/MgO Hall bar structure device. (a-b) Dependence of the anomalous Hall resistance $R_{\text {Hall }}$ on the in-plane magnetic field $H_{\mathrm{x}}$ at room temperature under different dc biases of $I_{\mathrm{x}}=18 \mathrm{~mA}$ and $I_{\mathrm{x}}=-18 \mathrm{~mA}$, respectively. (c-d) Current-induced magnetization switching at room temperature in the presence of constant in-plane magnetic fields of $H_{\mathrm{x}}=1000$ Oe and $H_{\mathrm{x}}=-1000$ Oe, respectively. 